\title{
Enriquecimento de floresta secundária com cedro-rosa (Cedrela odorata L.) e sumaúma (Ceiba pentandra (L.) Gaertn.), em Alta Floresta (MT)
}

\author{
Enrichment of secondary forest with cedro-rosa (Cedrela odorata L.) and \\ sumaúma (Ceiba pentandra (L.) Gaertn.), in Alta Floresta (MT)
}

\author{
Rubens Marques Rondon Neto'(*) \\ Carielen Alves Lage ${ }^{2}$ \\ Franciele Bilibio² \\ Aleandro Rodrigues dos Santos ${ }^{2}$
}

\section{Resumo}

O presente trabalho teve como objetivo avaliar o comportamento silvicultural de cedro-rosa (Cedrela odorata L.) e sumaúma (Ceiba pentandra (L.) Gaertn.) em floresta secundária após trinta meses de plantio, em Alta Floresta (MT), a fim de selecionar espécies florestais para o enriquecimento de florestas alteradas. O experimento foi instalado no sub-bosque de um fragmento de Floresta Ombrófila Aberta, contendo dois tratamentos (espécies florestais), constituído por quatro repetições de 25 indivíduos em cada uma, plantadas no espaçamento $3 \times 3 \mathrm{~m}$, no delineamento de blocos ao acaso. Os parâmetros avaliados foram: sobrevivência, altura total e diâmetro do tronco, aos 12, 18, 24 e 30 meses de idade. Os incrementos médios anuais em altura e diâmetro do tronco de Cedrela odorata e Ceiba pentandra foram de 1,0 m e 1,8 cm e 1,28 m e 2,2 cm, até os trinta meses de idade, respectivamente. As duas espécies apresentaram baixos níveis de mortalidade, sendo indicadas para recuperação de florestas alteradas, quando plantadas na forma de enriquecimento.

Palavras-chave: restauração florestal; espécie florestal; regeneração florestal.

\section{Abstract}

The present paper had as objective to evaluate the silvicultural behavior of the cedro-rosa (Cedrela odorata L.) and sumaúma (Ceiba pentandra (L.) Gaertn.)

I Dr.; Engenheiro Florestal; Professor Adjunto do Departamento de Engenharia Florestal da Universidade do Estado de Mato Grosso, UNEMAT, Campus de Alta Floresta; Endereço: Rodovia MT 208 - km 208, s/ no, 78.580-000, Alta Floresta, Mato Grosso, Brasil; E-mail: rubensrondon@yahoo.com.br (*) Autor para correspondência.

2 Engenheiros Florestais Autônomos. E-mail's: cari_elen@hotmail.com - fran_bilibio@hotmail.com aleandro.floresta@hotmail.com

Recebido para publicação em I8/05/2010 e aceito em 26/04/2011

Ambiência Guarapuava (PR) v.7 n.I p. 103 - $109 \quad$ Jan./Abr. 201। ISSN I808 - 025 I

DOI: 10.5777/ambiencia.2011.01.08 
in secondary forest after 30 months of plantation, in Alta Floresta/MT (Brazil), aiming to select forests species to enrichment of altered forest. The experiment was installed on the understory of a fragment Open Ombrophylous Forest, containing two treatments (forest species), constituted by four repetitions of 25 individuals in each, planted in the spacing $3 \times 3 \mathrm{~m}$, in randomized blocks desing. The parameters evaluated were: survival, total height and diameter of the trunk, at ages $12,18,24$ and 30 months. The mean annual increments in height and trunk diameter of Cedrela odorata and Ceiba pentandra were $1.0 \mathrm{~m}$ and $1.8 \mathrm{~cm}$ and $1.28 \mathrm{~cm}$ and 2.2, up to 30 months of age, respectively. The two species had low mortality, being indicated for restoration of forests changed when planted in the form of enrichment.

Key words: forest restauration; forests species; forest regeneration.

\section{Introdução}

O Brasil apresenta uma das maiores biodiversidades do mundo, tendo a região amazônica com uma grande diversidade de espécies florestais, no entanto, a falta de uma consciência ecológica e a exploração irracional tem ocasionado prejuízos irreparáveis para a floresta. Algumas dessas florestas já não respondem mais a tratamentos silviculturais que incentivem a regeneração natural, devendo ser substituídas por florestas de regeneração artificial, através do uso da semeadura direta ou plantio de mudas, para assegurar sua produtividade e continuidade (ALVARENGA, 2004).

Diante do crescente avanço da fronteira agropecuária, as áreas com cobertura florestal foram e ainda continuam sendo substituídas por cultivos agrícolas e pastagens, as quais, muitas vezes, são abandonadas após terem suas capacidades produtivas reduzidas, promovendo o surgimento das florestas secundárias. Em muitos desses locais, a sucessão florestal pode ser extremamente lenta, necessitando de aplicação de técnicas alternativas para acelerar o processo sucessional como o plantio de enriquecimento, que pode ser realizado por meio de sementes ou mudas, plantadas em faixa abertas na floresta ou em trilhas de arrastes deixadas após as atividades de exploração da madeira.

Atualmente, o plantio de mudas de espécies em diferentes grupos sucessionais tem sido o método mais utilizado em estudos de revegetação de áreas alteradas. Segundo Kageyama et al. (2003), o enriquecimento consiste em reintroduzir, num remanescente de florestas degradadas, espécies que não ocorrem mais na área em função da exploração ou do processo sucessional em que se encontra o fragmento de floresta a ser recuperado.

Sendo assim, este estudo teve como objetivo avaliar o comportamento silvicultural de cedro-rosa (Cedrela odorata L.) e sumaúma (Ceiba pentandra L.) Gaertn em floresta secundária após 30 meses de plantio, no município de Alta Floresta (MT), a fim de selecionar espécies florestais para o enriquecimento de florestas alteradas.

\section{Material e métodos}

\section{Área de estudo}

O presente estudo foi desenvolvido em um remanescente florestal secundário de Floresta Ombrófila Aberta, situada 
no município de Alta Floresta (MT), na coordenada geográfica 090 50' 53" latitude Sul, 56 07'49" longitude Oeste, com aproximadamente $264 \mathrm{~m}$ de altitude em relação ao nível do mar. A área total do fragmento florestal é de 6,15 ha, no entanto, o experimento ocupou apenas 0,18 ha.

O clima da região é do tipo Awi pela classificação de Köppen, com clima tropical chuvoso e nítida estação seca. A pluviosidade pode atingir médias muito elevadas, estando entre 2.500 a $2.750 \mathrm{~mm}$, com intensidade máxima em janeiro, fevereiro e março. A temperatura varia entre 20 a $38^{\circ} \mathrm{C}$, sendo a média de $26{ }^{\circ} \mathrm{C}$ (FERREIRA, 2001).

O solo predominante na região é classificado como Latossolo Vermelhoamarelo e relevo suave ondulado (MOREIRA e VASCONCELOS, 2007). As características químicas do solo na profundidade de 0 a $20 \mathrm{~cm}$ da área experimental, com as respectivas interpretações segundo Alvarez et al.(1999), são: $\mathrm{pH}\left(\mathrm{H}_{2} \mathrm{O}\right)=5,8$ (média) e $\mathrm{pH}\left(\mathrm{CaCl}_{2}\right)$ $=5,1$ (baixo); $\mathrm{P}=3,6$ (baixo) e $\mathrm{K}=61 \mathrm{mg}$ $\mathrm{dm}^{-3}$ (médio); $\mathrm{Ca}=2,25$ (médio), $\mathrm{Mg}=0,69$ (médio), $\mathrm{S}=3,1$ (baixo), $\mathrm{Al}=0,1$ (muito baixo), $\mathrm{H}=2,02$ e $\mathrm{H}+\mathrm{Al}=2,03$ (cmolc $\left.\mathrm{dm}^{-3}\right)$ (baixo), M.O. $=16\left(\mathrm{~g} \mathrm{dm}^{-3}\right), \mathrm{CTC}=$ $51\left(\mathrm{cmol} \mathrm{dm}^{-3}\right)$ (muito bom), $\mathrm{V}_{1}=60,0 \%$ (bom). A vegetação original de Floresta Ombrófila Aberta existente na área de estudo sofreu cortes seletivos para a extração de madeira de interesse comercial até o ano de 2003. No entanto, a partir desse período, a área vem sendo mantida sob proteção, evitando a derrubada de árvores e presença de queimadas. As duas espécies florestais estudadas ocorrem naturalmente nesse tipo de formação florestal da região.

\section{Instalação do experimento}

O experimento foi instalado no subbosque do fragmento da floresta secundária, contendo dois tratamentos, representados pelas espécies florestais cedro-rosa (Cedrela odorata) e sumaúma (Ceiba pentandra). Cada tratamento foi constituído por quatro parcelas de 25 indivíduos em cada uma, plantadas no espaçamento $3 \times 3 \mathrm{~m}$, no delineamento em blocos casualizados.

As mudas utilizadas para o plantio foram produzidas em sacos plásticos com dimensões de $15 \times 30 \mathrm{~cm}$, com alturas médias de 12,4 e 39,6 cm para Cedrela odorata e Ceiba pentandra, respectivamente. O plantio em covas de $30 \times 30 \times 40 \mathrm{~cm}$ foi realizado em novembro de 2005, coincidindo com o início do período de chuvas na região. Foram feitos tratos silviculturais como podas nos indivíduos quando necessário, além de tratos culturais como coroamento manual de 0,6 $\mathrm{m}$ de raio, roçadas nas entrelinhas e corte de cipós a cada dois meses. Não foi feito nenhum tipo de adubação nas mudas plantadas.

\section{Coleta e análise de dados}

Os parâmetros avaliados aos 12 , 18, 24 e 30 meses após o plantio foram: sobrevivência, altura total e diâmetro do caule a $15 \mathrm{~cm}$ do solo $\left(\mathrm{DAS}_{15}\right)$, medindo todos os indivíduos dentro de cada parcela. Para mensuração do diâmetro $(\mathrm{cm})$, foi utilizado paquímetro e para a altura $(\mathrm{m})$ utilizou-se trena. Foram feitas análise de variância e comparação de médias pelo teste de Tukey a 5\% de probabilidade, sendo utilizado o programa SISVAR para as três variáveis analisadas (FERREIRA, 2003). 


\section{Resultados e discussão}

\section{Sobrevivência}

Os valores de sobrevivência de Cedrela odorata até os trinta meses de avaliação são considerados altos, indicando que, até este momento, os indivíduos plantados não foram mortos pelo ataque da broca-do-cedro (Hypsipyla grandella Zeller, Lepidoptera: Pyralidae) (Tabela 1). Conforme Lamprecht (1990), o repetido ataque dessa praga acarreta a morte das árvores, ou pelo menos sua deformação, conferindo porte arbustivo.

Resultados inferiores foram encontrados por Martins et al. (1990) para Cedrela fissilis, em áreas em fase de regeneração natural anteriormente ocupadas com culturas agrícolas, em Maringá (PR), obtendo 35,9 e $33,7 \%$ de sobrevivência aos plantios puros feitos em áreas sem cobertura florestal com sete anos de idade, plantados no espaçamento $3 \times 4 \mathrm{~m}$, no município de Ouro Preto do Oeste/RO. Ainda nesse mesmo município, Lima et al. (1999) verificaram, em áreas desmatadas, $100 \%$ de sobrevivência aos 18 meses de idade, o que foi atribuído à elevada fertilidade natural da área, apresentando altos índices de fósforo e potássio no solo.

\section{Crescimento em altura}

Quanto ao crescimento em altura total verificou-se que, na primeira avaliação (12 meses), ocorreu diferença estatística significativa entre ambas as espécies, porém nas demais avaliações (18, 24 e 30 meses) não foram estatisticamente diferentes entre si (Tabela 2). No entanto, o crescimento

Tabela I. Sobrevivência (\%) de cedro-rosa (Cedrela odorata) e sumaúma (Ceiba pentandra), plantadas na forma de enriquecimento em uma Floresta Ombrófila Aberta secundária, em Alta Floresta (MT)

\begin{tabular}{lllll}
\hline \multirow{2}{*}{ Espécie } & \multicolumn{4}{c}{ Época de avaliação (meses) } \\
\cline { 2 - 5 } & 12 & 18 & 24 & 30 \\
\hline Cedrela odorata & $99 \mathrm{a}$ & $99 \mathrm{a}$ & $99 \mathrm{a}$ & $98 \mathrm{a}$ \\
Ceiba pentandra & $99 \mathrm{a}$ & $98 \mathrm{a}$ & $98 \mathrm{a}$ & $98 \mathrm{a}$ \\
\hline
\end{tabular}

Nota: Colunas seguidas das mesmas letras não diferem estatisticamente pelo teste de Tukey a $5 \%$ probabilidade.

24 e 60 meses após o plantio. Dessa forma, Carvalho (1994) recomendou que o plantio de espécies do gênero Cedrela deve ser feito na forma de enriquecimento, pois altas densidades de indivíduos podem favorecer o ataque de Hypsipyla grandella e o valor comercial da árvore ficar comprometido.

As taxas de sobrevivência da Ceiba pentandra são consideradas altas até o último período avaliado, demonstrando adaptação às condições ambientais de plantio. Altos índices de sobrevivência também foram encontrados por Vieira et al. (2007) em de Ceiba pentandra foi superior ao Cedrela odorata em 57,6\% aos 12 meses, 66,6\% aos dezoito meses, $79,5 \%$ aos 24 meses e $78,2 \%$ aos 30 meses de idade. Os ataques da Hypsipyla grandella nas plantas jovens de Cedrela odorata provocaram a morte da gema apical e diminuição da área de copas, o que pode ter contribuído na diminuição do crescimento em altura.

Os incrementos médios anuais em altura de Cedrela odorata e Ceiba pentandra foram de 1,00 e 1,28 $\mathrm{m}$ até os trinta meses de idade, respectivamente. Valor inferior de 
Tabela 2. Crescimento em altura total $(\mathrm{m})$ de cedro-rosa (Cedrela odorata) e sumaúma (Ceiba pentandra), plantadas na forma de enriquecimento em Floresta Ombrófila Aberta secundária, em Alta Floresta (MT)

\begin{tabular}{lcccc}
\hline \multirow{2}{*}{ Espécie } & \multicolumn{4}{c}{ Época de avaliação (meses) } \\
\cline { 2 - 5 } & 12 & 18 & 24 & 30 \\
\hline Cedrela odorata & $1,09 \mathrm{a}$ & $1,60 \mathrm{a}$ & $2,06 \mathrm{a}$ & $2,51 \mathrm{a}$ \\
Ceiba pentandra & $1,89 \mathrm{~b}$ & $2,40 \mathrm{a}$ & $2,59 \mathrm{a}$ & $3,21 \mathrm{a}$ \\
\hline
\end{tabular}

Nota: Colunas seguidas das mesmas letras na não diferem estatisticamente pelo teste de Tukey a $5 \%$ probabilidade.

crescimento em altura para Cedrela odorata foi obtido por Paiva e Poggiani (2000), encontrando uma média de $0,50 \mathrm{~m}$ aos doze meses de idade e sendo atacadas por Hypsipyla grandella, quando plantado na forma de enriquecimento de um fragmento florestal, no município de Guará/SP. Em condições de plantio de Cedrela odorata em pleno sol e em linhas na capoeira, Souza et al. (2010) obtiveram 0,9 e 1,5 m de incremento médio anual em altura. Essas diferenças de taxas de crescimento em altura podem ser atribuídas aos níveis de sombreamento das florestas e das qualidades dos sítios, o que está em acordo com Lamprecht (1990), o qual classifica Cedrela odorata quanto ao grupo sucessional de pioneira, conferindo necessidade de luz em todas as fases de seu crescimento.

Lamprecht (1990) comentou que a Ceiba pentandra apresenta crescimento rápido, nos primeiros 10 anos taxas de incremento em altura de 1,2 m.ano ${ }^{-1}$. Em Boa Vista/RR, Arco-Verde e Schwengber (2003) observaram que, a céu aberto, Ceiba pentandra teve um incremento médio anual em altura de 1,67 $\mathrm{m}$ até os trinta meses. Portanto, estas taxas de crescimento são superiores às encontradas no presente trabalho, o que pode ser atribuído ao fato de Ceiba pentandra pertencer ao grupo sucessional de espécie secundária inicial a secundária tardia (LORENZI, 2000), necessitando de uma maior intensidade de luz em sua fase inicial de crescimento. Em Manuas/AM, Souza et al. (2010) constaram que, dentre 10 e 14 espécies florestais plantadas em pleno sol e em faixas na capoeira, aos seis anos de idade Ceiba pentandra foi a que apresentou o melhor crescimento e adaptação.

\section{Crescimento em diâmetro}

Os valores de crescimento em diâmetro das duas espécies florestais estudadas apresentaram diferenças estatísticas significativas apenas na primeira avaliação, aos doze meses de idade (Tabela 3). No entanto, o crescimento em diâmetro de Ceiba pentandra novamente foi superior ao de Cedrela odorata em 60,0\% aos 12 meses,

Tabela 3. Crescimento em diâmetro do fuste a $15 \mathrm{~cm}$ de altura do solo $(\mathrm{cm})$ de cedro-rosa (Cedrela odorata) e sumaúma (Ceiba pentandra), plantadas na forma de enriquecimento em Floresta Ombrófila Aberta secundária, em Alta Floresta (MT)

\begin{tabular}{|c|c|c|c|c|}
\hline \multirow{2}{*}{ Espécie } & \multicolumn{4}{|c|}{ Época de avaliação (meses) } \\
\hline & 12 & 18 & 24 & 30 \\
\hline Cedrela odorata & $1,50 \mathrm{a}$ & $3,50 \mathrm{a}$ & $3,50 \mathrm{a}$ & $4,50 \mathrm{a}$ \\
\hline Ceiba pentandra & $2,50 \mathrm{~b}$ & $4,50 \mathrm{a}$ & $4,50 \mathrm{a}$ & $5,50 \mathrm{a}$ \\
\hline
\end{tabular}

Nota: Colunas seguidas das mesmas letras não diferem estatisticamente pelo teste de Tukey a 5\% probabilidade. 
$77,7 \%$ aos dezoito meses, $77,7 \%$ aos 24 meses e 81,8 aos trinta meses de idade. O maior desenvolvimento dos indivíduos de Ceiba pentandra em condições de enriquecimento de florestas secundárias, deve-se à maior adaptabilidade da espécie em condições de competição por luz. Tal fato está em acordo com Lorenzi (2000), o qual classifica Ceiba pentandra como uma espécie secundária inicial a tardia, portanto, tolera ambientes sombreados na fase inicial de crescimento.

$O$ incremento médio anual em diâmetro do tronco para Cedrela odorata e Ceiba pentandra foi de 1,8 e 2,2 cm até os trinta meses, respectivamente. Lamprecht (1990) relatou que indivíduos de Ceiba pentandra apresentaram, nos primeiros 10 anos de idade, taxas de incremento em diâmetro de 3 a $4 \mathrm{~cm}$.ano ${ }^{-1}$. Arco-Verde e Schwengber (2003) observaram que, a céu aberto, em Boa Vista/RR, essa espécie apresentou uma média de incremento em diâmetro de $3,47 \mathrm{~cm}$ aos trinta meses de idade. Tal valor é superior ao encontrado no presente trabalho, devido haver uma maior competição por luz dos indivíduos de Ceiba pentandra plantados na forma de enriquecimento no sub-bosque do fragmento florestal.

\section{Conclusão}

As espécies cedro-rosa (Cedrela odorata) e sumaúma (Ceiba pentandra) apresentaram desenvolvimento satisfatório quando plantadas no sub-bosque de floresta secundária na forma de enriquecimento, demonstrando certa adaptação ao ambiente de plantio. O crescimento de Ceiba pentandra foi superior ao de Cedrela odorata em altura e diâmetro até os trinta meses de idade. Ambas as espécies apresentaram baixos níveis de mortalidade, portanto, até o momento avaliado são indicadas para recuperação de florestas alteradas na forma de enriquecimento.

\section{Referências}

ALVAREZ, V.H.; NOVAIS, R. F.; BARROS, N. F. de; CANTARUTTI, R. B.; LOPES, A. S. Interpretação dos resultados das análises de solos. In: RIBEIRO, A. C.; GUIMARAES, P. T. G.; ALVAREZ V., V. H. (Ed.). Recomendações para o uso de corretivos e fertilizantes em Minas Gerais: $5^{a}$ aproximação. Viçosa: Comissão de Fertilidade do Solo do Estado de Minas Gerais, 1999. p.25-32.

ALVARENGA, A. P. Avaliação inicial de recuperação de mata ciliar em nascentes. 2004. 175f. Dissertação (Mestrado em Engenharia Florestal) - Universidade Federal de Lavras, Lavras, Minas Gerais, 2004.

ARCO-VERDE, M. F; SCHWENGBER, D. R. Avaliação silvicultural de espécies florestais no estado de Roraima. In: ENCONTRO DE PESQUISA E INICIAÇÃO CIENTÍFICA DA UNIVERSIDADE FEDERAL DE RORAIMA, 1., 2002, Boa Vista. Anais... Boa Vista: UFRR, 2002.

CARVALHO, P. E. R. Espécies florestais brasileiras: recomendações silviculturais, potencialidades e uso da madeira. Brasília: EMBRAPA/CNPF, 1994. 640p. 
FERREIRA, D. F. SISVAR - Sistema para análise de variância de dados balanceados: versão 4.3. Lavras: DEX/UFLA, 2003.

FERREIRA, J. C. V. Mato Grosso e seus municípios. Cuiabá: Secretaria de Estado da Educação, 2001.365p.

KAGEYAMA, P. Y.; SEBBEN, A. M.; RIBAS, L. A.; GANDARA, F. B.; CASTELLEN, M.; PERECIN, M. B.; VENCOVSKY, R. Diversidade genética em espécies arbórea tropicais de diferentes estágios sucessionais por marcadores genéticos. Scientia Forestalis, Piracicaba, n.64, p.93-107, 2003.

LAMPRECHT, H. Silvicultura nos trópicos: ecossistemas florestais e respectivas espécies arbóreas - possibilidade e métodos de aproveitamento sustentado. Eschborn: GTZ, 1990. $343 p$.

LIMA, R. M. B.; MARQUES, L. C. T.; OLIVEIRA, J. M. F.; KALIL FILHO, A. N.; AZEVEDO, C. P. de; GARCIA, L. C.; Teixeira, W. G. Zoneamento edafo-climático para plantio de espécies florestais de rápido crescimento na Amazônia. In: BRASIL. Ministério da Ciência e Tecnologia. (Org.). PPG-7 C\&T Resultados (Fase Emergencial e Fase 1). Brasília: 1999. p.310-331.

LORENZI, H. Árvores brasileiras: manual de identificação e cultivo de plantas arbóreas nativas do Brasil. 3. Ed. Nova Odessa: Instituto Plantarum, 2000. v.1, p.51 e 156.

MARTINS, S. S; TAKAHASHI, L. Y; BORGES, R. C. G. Desenvolvimento de algumas espécies florestais nativas em plantio de enriquecimento. Revista Silvicultura, São Paulo, v.2, n.42, p.239-242, 1990.

MOREIRA, M. L. C; VASCONCELOS, T. N. N. Mato Grosso: solos e paisagens. Cuiabá: SEPLAN/MT, 2007.272p.

PAIVA, A.V.; POGGIANI, F. Crescimento de mudas de espécies arbóreas nativas plantadas no sub-bosque de um fragmento florestal. Scientia Forestalis, Piracicaba, n.57, p.141-151, 2000 .

SOUZA, C. R. de.; AZEVEDO, C. P. de; LIMA, R. M.; ROSSI, L. M. B. Comportamento de espécies florestais em plantios a pleno sol e em faixas de enriquecimento de capoeira na Amazônia. Acta Amazônica, Manaus, v.40, n.1, p.127-134, 2010.

VIEIRA, A. H.; ROCHA, R. B.; GAMA, M. M. B.; LOCATELLI, M. Desempenho de sumaúma (Ceiba pentandra Gaertn) em plantio adensado no Estado de Rondônia. Porto Velho: Embrapa - Rondônia (Circular Técnica 95), 2007. 4p. 